\title{
Energy Harvesting from Drops Impacting onto Charged Surfaces
}

\author{
Hao Wu $\odot,{ }^{1,2, *}$ Niels Mendel $\odot,{ }^{1}$ Dirk van den Ende, ${ }^{1}$ Guofu Zhou, ${ }^{2, \dagger}$ and Frieder Mugele ${ }^{1, \$}$ \\ ${ }^{1}$ Physics of Complex Fluids, Faculty of Science and Technology, MESA+ Institute for Nanotechnology, University of Twente, \\ Enschede 7500AE, The Netherlands \\ ${ }^{2}$ Guangdong Provincial Key Laboratory of Optical Information Materials and Technology \& Institute of Electronic Paper Displays, \\ South China Academy of Advanced Optoelectronics, South China Normal University, \\ Guangzhou 510006, People's Republic of China
}

(Received 6 March 2020; revised 15 May 2020; accepted 25 June 2020; published 12 August 2020)

\begin{abstract}
We use a combination of high-speed video imaging and electrical measurements to study the direct conversion of the impact energy of water drops falling onto an electrically precharged solid surface into electrical energy. Systematic experiments at variable impact conditions (initial height; impact location relative to electrodes) and electrical parameters (surface charge density; external circuit resistance; fluid conductivity) allow us to describe the electrical response quantitatively without any fit parameters based on the evolution of the drop-substrate interfacial area. We derive a scaling law for the energy harvested by such "nanogenerators" and find that optimum efficiency is achieved by matching the timescales of the external electrical energy harvesting circuit and the hydrodynamic spreading process.
\end{abstract}

DOI: 10.1103/PhysRevLett.125.078301

The impact of liquid drops onto solid surfaces leads to conversion of kinetic energy of directed drop motion into various forms of energy including surface energy, vibrational energy, heat, and-under suitable conditionselectrical energy [1-8]. The latter has attracted substantial attention in recent years for its potential to directly convert energy from random environmental drop and contact line motion as in rainfall, spray, and surface waves to electrical energy [6,7,9-11]. Numerous configurations of such "electrical nanogenerators" (ENGs) have been invented using one or two electrodes on a substrate typically connected to an external electric energy harvesting circuit with a load resistor $[9,12]$. The electrodes are covered by a hydrophobic dielectric layer that typically carries some permanent surface charges. The drop acts as an additional electrode in the circuit that translates and deforms upon hitting the surface. Doing so, it changes the capacitive coupling between the various electrodes in the system and thereby, in conjunction with the permanent charges, induces the desired electrical currents for energy harvesting. On a conceptual level, the process is thus the inverse of electrowetting, where electrical signals are used to stimulate the mechanical deformation and displacement of drops [13-15]. However, unlike electrowetting where charges usually equilibrate much faster than the liquid, electrical and fluid dynamic processes in ENGs are more interwoven and their interplay has yet to be disentangled. In many ENGs, the water drops also fulfill a second role: in addition to providing the required initial mechanical energy, the impact process is also responsible for the generation of the trapped surface charge on the hydrophobic surface $[6,9,12]$. However, like other triboelectric charging mechanisms that have been explored, this process is notoriously difficult to control and dependent on details of materials and process conditions [16-18]. The poor stability of surface charges generated by drop impact, tribocharging, and hydrophobicwater contact [19-24] compromises the performance and stability of possible devices and has also hampered the development of a quantitative model of the energy conversion process, which is needed to provide guidelines for further improvements of the technology.

The purpose of this Letter is to develop a physical picture and quantitative model of the electrical response caused by the impact of a drop onto an ENG surface based on simultaneous high-speed video imaging and high-speed electrical current measurements. Making use of two recent developments in the literature, namely an improved electrode geometry [6,25] and a robust charging mechanism [25-27], we systematically screen a wide range of experimental parameters to validate our model and to derive a scaling relation for the maximum harvested energy. The latter is given by the product of a purely electrostatic (capacitive) contribution and a nondimensional integral that is controlled by fluid dynamic and other process parameters.

In our experiments, we released millimeter-sized drops from a height $h$ of 3 to $18 \mathrm{~cm}$ onto two types of horizontal or slightly inclined surfaces [Fig. 1(a)]: for simultaneous high-speed video imaging and electrical measurements we used transparent indium-tin-oxide-covered glass slides; for all-electrical measurements, highly doped Si wafers with a $300 \mathrm{~nm}$ oxide layer. In both cases, the surfaces were coated with an $800 \mathrm{~nm}$ to $1000 \mathrm{~nm}$ thick film of an amorphous fluoropolymer (AFP; Teflon ${ }^{\circledR}$ AF 1600) [28]. The polymer films were electrically charged prior to the experiment 

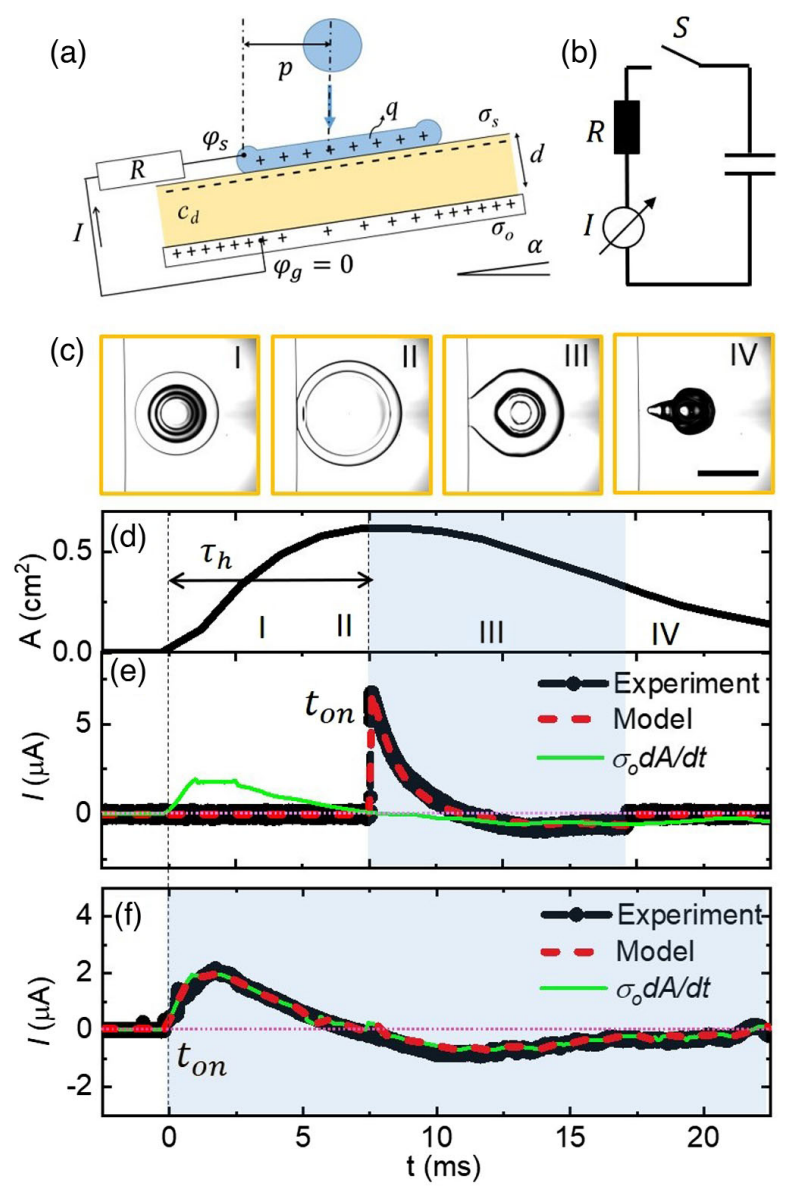

FIG. 1. (a) Experimental setup (not to scale; $d \ll$ drop radius; $\alpha=0, \ldots, 30^{\circ}$ ). (b) Simplified equivalent circuit (for full circuit, see Supplemental Material [28], Sec. III). (c) Bottom view snapshots for various stages of impact through transparent substrates. Scale bar: $5 \mathrm{~mm}$ (off-wire impact: $p=a_{\max }$ ). (d) Drop-substrate contact area vs time. (e) Simultaneously recorded current response ( $\left.p=a_{\max } ; R=810 \mathrm{k} \Omega ; \sigma_{o}=0.12 \mathrm{mC} / \mathrm{m}^{2}\right)$. (f) Current for "onwire" impact ( $p=0 ; R=0 \Omega ; \sigma_{o}=0.12 \mathrm{mC} / \mathrm{m}^{2}$ ). Blue region: time interval of drop-wire contact. Black symbols: experimental data; red curve: model current; pink dashed line: $I=0$. All data: $\alpha=0^{\circ}$; drop volume: $33 \mu \mathrm{L} ; 100 \mathrm{mM} \mathrm{NaCl}$.

by either dropping more than 500 identical drops onto the surface (indium-tin-oxide samples; surface charge $\sigma_{s}=$ $\left.-0.12, \ldots, 0.16 \mathrm{mC} / \mathrm{m}^{2}\right)$ or by electrowetting-assisted charge injection (EWCI) [26] for the $\mathrm{Si}$ wafers $\left(\sigma_{s}=\right.$ $\left.-0.07, \ldots,-0.35 \mathrm{mC} / \mathrm{m}^{2}\right)$; see Supplemental Material [28], Sec. I for a description of the independent surface charge measurements. The electrode on the substrate is connected via an external load resistor $R$ to a thin Pt wire that is mounted parallel to the substrate using spacers at a distance of 0.05 to $0.1 \mathrm{~mm}$. The current through the resistor is monitored using a fast transimpedance amplifier in combination with a digital storage oscilloscope (see Supplemental Material [28], Sec. II). Unless specified otherwise, all experiments were conducted with $100 \mathrm{mM}$ aqueous solutions of $\mathrm{NaCl}$.
Drops hit the surface (time $t=0$ ) at a distance $p$ from the wire, start to spread, and assume a pancake structure with a pronounced rim, Fig. 1. We extract the dropsubstrate area $A(t)$ from the bottom view images [32] (see Supplemental Material [28], Sec. II) and denote the time $\tau_{h}$ between the moment of impact and maximum spreading with $A\left(t=\tau_{h}\right)=A_{\max }$ as characteristic hydrodynamic time [ $\approx 7 \mathrm{~ms}$ in Fig. 1(d)]. $\tau_{h}$ is largely determined by Rayleigh's inertia-capillary timescale $\sqrt{\rho a^{3} / \gamma}$, where $\rho$, $a$, and $\gamma$ are the density, radius, and surface tension $[2,4]$. The maximum spreading radius $a_{\max }$ is controlled by the Weber number $\mathrm{We}=\left(\rho v^{2} a / \gamma\right)=13, \ldots, 77$ upon impact (impact speed: $v=0.71, \ldots, 1.89 \mathrm{~m} / \mathrm{s}$ ). At a $p$-dependent time $t_{\mathrm{on}}$, the drop touches the wire and induces a current peak. Subsequently, the current decreases from its initial peak value $I_{o}$ within an electrical relaxation time $\tau_{\mathrm{el}} \approx 2 \mathrm{~ms}$ in Fig. 1(e) and eventually follows a much slower evolution proportional to $d A / d t$ [green line in Fig. 1(e)]. At long times, the drop recedes, detaches from the wire $\left(t=t_{\text {off }}\right)$ and eventually either bounces off or rolls down the slightly inclined surface (see Videos S1-S5). The current vanishes at the moment $t=t_{\text {off }}$. $t_{\text {on }}$ and $t_{\text {off }}$ can be controlled by varying the impact parameter $p$ and the tilt angle $\alpha=0^{\circ}, \ldots, 30^{\circ}$. Drops hit the wire earlier for smaller values of $p$. This leaves the height of the current peak unaffected but reduces the electrical relaxation time (Supplemental Material [28], Fig. S7). Only upon direct on-wire impact $(p=0)$ the current response is qualitatively different and does not display any peak. Instead, in the case of $\tau_{\text {el }} \ll \tau_{h}$, it follows a smooth curve $I(t)=\sigma_{o} d A / d t$, where $\sigma_{o}=-\sigma_{s}$ is the charge density required to compensate the surface charge [Fig. 1(f)]. Exploring the dependence of $I(t)$ on various electrical parameters, we find that $I_{o}$ increases with decreasing $R$ and with increasing surface charge. Yet, it is independent of $h$ and of $A_{\max }$. The response in Fig. 2 resembles the characteristic exponential discharging of a capacitor in an $R C$ circuit. Ignoring negligible elements such as electric double layers (see Supplemental Material [28], Sec. III), we represent the system by an equivalent $R C$ circuit with the peculiarity of an explicitly time-dependent capacitance $C(t)=c_{d} A(t)$, Fig. 1(b). Here, $c_{d}=$ $\left(\epsilon_{0} \epsilon_{d} / d\right) \approx 10^{-5} \mathrm{~F} / \mathrm{m}^{2} \quad\left(\epsilon_{0} \epsilon_{d}\right.$ : dielectric permittivity of the fluoropolymer layer) is the capacitance per unit area between drop and substrate. A second peculiarity of the system arises from the presence of the permanent surface charge $\sigma_{s}=-\sigma_{o}$, which implies that there is an intrinsic initial voltage $U_{s}=\sigma_{s} / c_{d}$ across the capacitor at $t \leq t_{\text {on }}$. At $t=t_{\mathrm{on}}$, the drop touches the wire and charge is transferred from the bottom electrode to the drop giving rise to the steep current increase [33]. As current flows, the capacitor is discharged and thus the electric potential on the dielectric surface $\varphi_{s}(t)=U_{s}+q(t) / C$ increases toward zero. Here, $q(t)=\int_{t_{\mathrm{on}}}^{t} I\left(t^{\prime}\right) d t^{\prime}$ is the total charge transferred between $t_{\mathrm{on}}$ and $t$. The resulting current is 
(a)

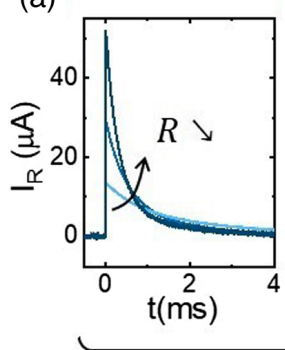

(b)

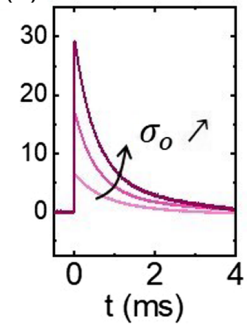

(c)

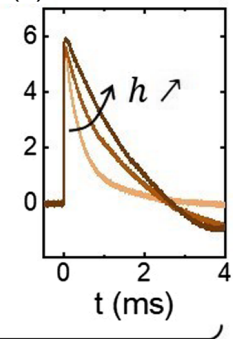

(d)

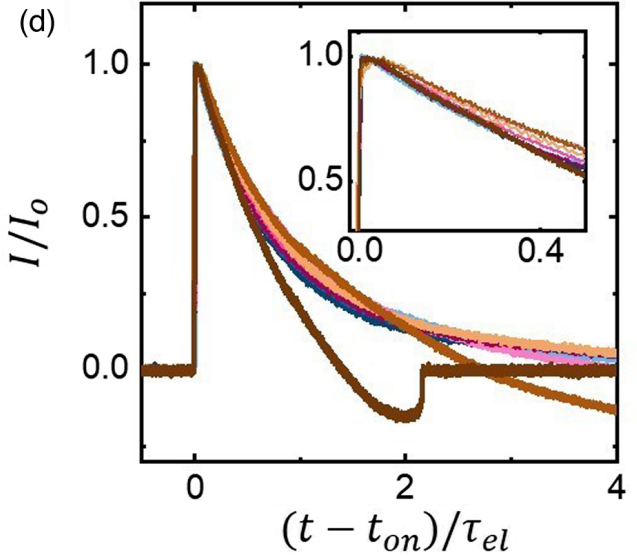

FIG. 2. Current response from drop impact with (a) variable $R \quad(0.47,0.81,1.651 .65 \mathrm{M} \Omega)$; fixed $\sigma_{o}=0.35 \mathrm{mC} / \mathrm{m}^{2}$; $h=43 \mathrm{~mm} ; p=a_{\max } ; \alpha=30^{\circ}$ (oxidized silicon samples); (b) variable charge density $\left(\sigma_{o}=0.07,0.18,0.35 \mathrm{mC} / \mathrm{m}^{2}\right)$; fixed $R=810 \mathrm{k} \Omega ; h=43 \mathrm{~mm} ; p=a_{\max } ; \alpha=30^{\circ} ; \mathrm{We}=46$ (oxidized silicon samples). (c) Varying impact height ( $h=30,90,180 \mathrm{~mm}$; $\mathrm{We}=13,39,77)$; fixed $\sigma_{o} \approx 0.1 \mathrm{mC} / \mathrm{m}^{2} ; R=810 \mathrm{k} \Omega ; p=a_{\max }$; $\alpha=0^{\circ}$ (transparent samples); Drop volume for (a) and (b) is $33 \mu \mathrm{L}$, for (c) it is $17 \mu \mathrm{L}$. (d) Nondimensional current response curve vs normalized time for all data from panels (a)-(c). Inset: enlarged view for $t-t_{\mathrm{on}} \ll \tau_{h}$.

$$
I(t)=\frac{d q}{d t}=\frac{1}{R c_{d}}\left(\sigma_{o}-\frac{q(t)}{A(t)}\right)
$$

Taking into account that $q\left(t_{\mathrm{on}}\right)=0$, it follows immediately for all off-wire impacts that the initial current is given by $I_{o}=\sigma_{o} / c_{d} R$, as seen in Figs. 2(a)-2(c). Using $A_{\max }$ as the characteristic area, we can introduce $q_{0}=\sigma_{o} A_{\max }$, $\tau_{\mathrm{el}}=R c_{d} A_{\max }$, and $\tau_{h}$ as characteristic charge, electrical relaxation time, and hydrodynamic spreading time, respectively, and rewrite Eq. (1) in dimensionless form as

$$
\frac{d \tilde{q}}{d \tilde{t}}=\frac{1}{\tilde{\tau}_{\mathrm{el}}}\left(1-\frac{\tilde{q}}{\tilde{A}}\right)
$$

Here, quantities with a tilde are measured in nondimensional units, namely $\tilde{q}=q / q_{0}, \quad \tilde{t}=t / \tau_{h}, \tilde{\tau}_{\text {el }}=\tau_{\text {el }} / \tau_{h}$, and $\tilde{A}=A / A_{\max }$. For $t-t_{\text {on }} \ll \tau_{h}$ and simultaneously $\tau_{\mathrm{el}} \ll \tau_{h}, \tilde{A}$ is approximately constant and $I(t)$ relaxes exponentially. Note, however, that the observed electrical relaxation time depends $C(t)=c_{d} A(t)$ and can therefore be much smaller than $\tau_{\mathrm{el}}$. For instance, the momentary electrical relaxation time immediately after hitting the wire is $\tau_{\mathrm{el}}^{\prime}=\left[A\left(t_{\mathrm{on}}\right) / A_{\mathrm{max}}\right] \tau_{\mathrm{el}}$, which vanishes for on-wire impacts. This explains the faster relaxation for impacts with $p<a_{\max }$ (Fig. S7). All data in Fig. 2 correspond to $p=a_{\max }$. Hence, for a short time after impact $\left[\left(t-t_{\text {on }}\right) \ll \tau_{\text {el }}\right]$ all data collapse upon plotting $I / I_{0}$ vs $\left(t-t_{\mathrm{on}}\right) / \tau_{\mathrm{el}}$, Fig. 2(d). At later stages, the current is affected by the variation of $A(t)$ and hence display dispersion. [This deviation is most pronounced for drops falling from variable initial height-Fig. 2(c) — which had a smaller volume than in Figs. 2(a) and 2(b) and hence also switch earlier from spreading to receding motion.]

For $\tau_{\mathrm{el}} \ll \tau_{h}$, the drop continues to spread or recede even after the initial current peak has relaxed. During that phase, the charge on the capacitor adiabatically follows the spreading dynamics such that the right-hand side of Eq. (2) always vanishes, i.e., $\tilde{q}(t)=\tilde{A}(t)$. This corresponds to the later stages of the current response in Figs. 1(e) and 1(f), for which we indeed find in dimensional units $\dot{q}(t)=\sigma_{o} \dot{A}(t)$. This also explains the reversal of the current as the drop recedes. The fact that the capacitor is completely discharged under these conditions can also be confirmed by integrating the experimental current curves from $t_{\text {on }}$ to $\tau_{h}$ for $\tau_{\mathrm{el}} \ll \tau_{h}-t_{\mathrm{on}}$. In this case, the total transferred charge is indeed found to be $Q_{\max }=\sigma_{o} A_{\max }$, see Fig. S9.

The complete current response for all times is obtained by numerically solving Eq. (2) with the initial condition $\tilde{q}\left(\tilde{t}_{\text {on }}\right)=0$ and using the experimental spreading curve $\tilde{A}(t)$ as input. The result [dashed lines in Figs. 1(e) and 1(f)] reproduces the experimental data without any fit parameter. Having achieved a complete understanding of the current response, we explored materials and process parameters to optimize the performance of the system. The data in Figs. 1 and 2 represent situations with $\tau_{\mathrm{el}}$ being a sizable fraction of $\tau_{h}$. As a consequence, the charge relaxation is faster than the drop spreading. Upon choosing $R$ even smaller $\left(\tau_{\mathrm{el}} \ll \tau_{h}\right)$, the initial charge relaxation-controlled current and the subsequent spreading-controlled current become completely separated in time. Since the total amount of charge transferred remains equal to $\sigma_{o} A_{\max }$, the current peaks become increasingly narrow and high. The spreading-controlled current following charge relaxation, follows exactly the same response curve as for on-wire impacts (Fig. S8a). For $\tau_{\mathrm{el}} \approx \tau_{h}$, the $I(t)$ curves for on-wire and off-wire impact become similar as the initial current peak decreases and becomes wider (Fig. S8b). For $\tau_{\text {el }} \gg \tau_{h}$, $I(t)$ vanishes because the electrical relaxation becomes so slow that no substantial charge relaxation takes place throughout the entire drop impact and rebounding process (data not shown).

According to the expression $I_{0}=\sigma_{o} / R c_{d}$ the peak current and power can be increased indefinitely by choosing sufficiently small load resistors. Indeed, the maximum 


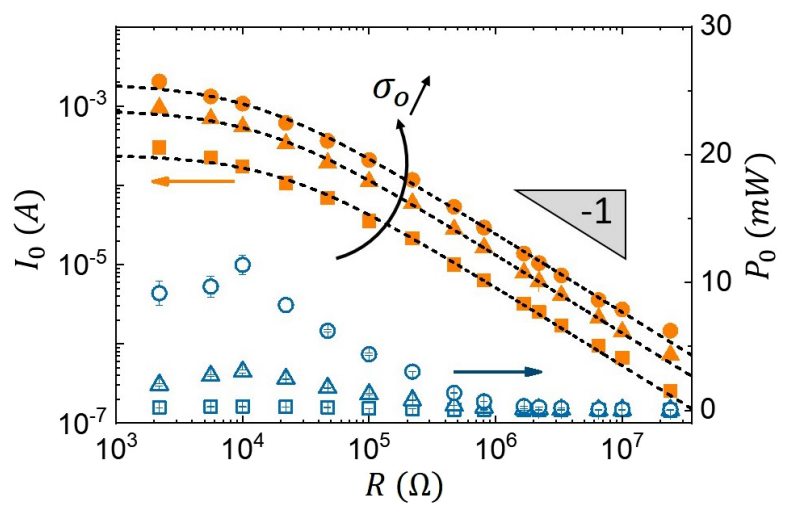

FIG. 3. Peak current and power vs load resistance for variable surface charge. (Squares: $\sigma_{o}=0.07 \mathrm{mC} / \mathrm{m}^{2}$; triangles: $0.18 \mathrm{mC} / \mathrm{m}^{2}$; circles: $0.35 \mathrm{mC} / \mathrm{m}^{2}$. Orange and closed symbols: current peak value; blue and open symbols: maximum power value; dashed line: model with $I_{0}=\sigma_{o} /\left[\left(R+R_{\mathrm{drop}}\right) c_{d}\right]$ including finite drop resistance; all data: drop volume: $33 \mu \mathrm{L} ; 100 \mathrm{mM}$ $\mathrm{NaCl}$ solution; $h=43 \mathrm{~mm} ; p=a_{\max } ; \alpha=30^{\circ}$.)

peak currents in our experiments $(\approx 2.2 \mathrm{~mA}$; Fig. 3) are achieved at for the lowest $R$ (and the highest $\sigma_{o}$ ). They exceed typical values in the literature (nanoampere to low microampere level) by several orders of magnitude and follow the expected $1 / R$ scaling, except for the lowest $R$. This improvement is largely owed to the direct contact of the Pt wire and the drop, which replaces the capacitive coupling across the dielectric layer of earlier ENG configurations that often contain dielectric layers on the electrodes [7,34].

Along with the peak current the instantaneous power $P(t)=R I(t)^{2}$ also reaches very high maximum values. Yet, $I_{0}$ saturates for low $R$, as shown in Fig. 3. Therefore, the maximum power is found for finite $R$. The deviation of $I_{0}$ from the $1 / R$ scaling can be explained by taking into account the finite conductivity of the drop. Experiments at variable salt concentration demonstrate that the deviation indeed shifts toward increasing $R$ with decreasing conductivity (Fig. S10). Upon including an additional resistor $R_{\text {drop }}$ in series with $R$, the linear scaling of $I_{0}$ with the inverse of the total resistance $R_{\mathrm{tot}}=R+R_{\text {drop }}$ is indeed recovered (Fig. S10). $R_{\text {drop }}$ is found to scale inversely with the conductivity of the electrolyte as expected.

Notwithstanding the focus on high peak current and power in the applied literature [6], the actual quantity of interest is the maximum harvested energy $E$ per drop, which is calculated by integrating the instantaneous power

$$
E=R \int_{t_{\mathrm{on}}}^{t_{\mathrm{off}}} I^{2}(t) d t=E_{0} F\left(\tilde{t}_{\mathrm{on}}, \tilde{\mathrm{t}}_{\mathrm{off}}, \tilde{\tau}_{\mathrm{el}},\left\{\alpha_{n}\right\}\right) .
$$

The right-hand side of the equation is obtained by writing current and time again in normalized units. Here, $E_{0}=\left(\sigma_{o}^{2} A_{\max } / c_{d}\right)$ is the characteristic energy scale.
$F=\int(d \tilde{q} / d \tilde{t})^{2} d \tilde{t}$ is a nondimensional function of dimensionless times $\tilde{t}_{\mathrm{on}}, \tilde{t}_{\mathrm{off}}$, and $\tilde{\tau}_{\mathrm{el}}$, and $\left\{\alpha_{n}\right\}$ is a set of parameters that describes $\tilde{A}(\tilde{t})$. These parameters are determined by the fluid dynamics of the impact process, see, e.g., [35], including tilt angle and impact parameter. $E_{0}$ is twice the electrostatic energy of a parallel plate capacitor $C=c_{d} A_{\max }$ with charge $q_{0}$. This can be rationalized by realizing that the total charge $q_{0}$ relaxes from its initial separation $d$ on the capacitor to their final separation on the drop that is given by the electric double layer thickness of a few nanometers as it is transferred from the bottom electrode to the drop. As the drop recedes, the charges are separated again back to the original distance. During both phases, the electrical current dissipates energy in the load resistor. The role of the mechanical motion upon drop impact is thus to modulate the capacitance of the system in time, very similar to conventional energy harvesting system with vibrating capacitor plates $[36,37]$. In fact, the initial charge relaxation upon spreading converts electrical energy into (mechanical) surface energy due to electrowetting, leading to a more pronounced spreading as compared to an uncharged identical system. However, since $E_{0} / A_{\max }=$ $\sigma_{o}^{2} / c_{d} \ll \gamma$ in the present experiments, we were unable to detect substantial deviations of the spreading behavior. Any small correction that should be present in our data is implicitly included in the empirical profiles $A(t)$ that we use as an input for our electrical model.

Calculating the harvested energy from a large number of measurements for variable $\sigma, R$, and $t_{\mathrm{on}}$, we confirm indeed the scaling of Eq. (3) for both off-wire (here, $p=a_{\max }$, where $a_{\max }$ is the radius of the drop at maximal spreading), and on-wire impacts, Fig. 4. As expected, higher surface charge densities give rise to higher energy harvesting for all $p$ and $R$. Scaling $E$ by $E_{0}$ and $\tau_{\text {el }}$ by $\tau_{h}$ leads to universal energy harvesting characteristics that depend only on $p$. Independent of $\sigma_{o}$ and $p$, optimum energy harvesting is achieved for values of $R$ that correspond to $\tau_{\mathrm{el}} \approx \tau_{h}$. This is similar to the behavior of a regular $R C$ circuit driven by an external alternating current, where the dissipation is also maximum if the driving frequency matches the intrinsic relaxation time. While $E$ drops linearly for $\tau_{\text {el }} \ll \tau_{h}$ for onwire impacts, off-wire impacts display a much weaker dependence (caused by the resistance of the drop, $R_{\mathrm{drop}}$ ). This robustness for off-wire impact arises from the initial high current peak for $p>0$ that results in a quick release of the initial electrostatic energy in the capacitor. The inset of Fig. 4(b) shows calculated profiles of $F$ as a function of $\tilde{\tau}_{\text {el }}$ for a variety of values of $p$ (or equivalently $\tilde{t}_{\text {on }}$ ). (The slight decrease in $E$ in the main panels of Fig. 4 for $p=a_{\max }$ is caused by the finite value of $R_{\text {drop }}$ that is not included here.) For optimum conditions in our experiments, we achieved a maximum value $E \approx 0.4 \mu \mathrm{J}$ (Fig. 4). Given the initial gravitational energy of $\approx 14 \mu \mathrm{J}$, this corresponds to a conversion efficiency of $2.5 \%$, much higher than previous 

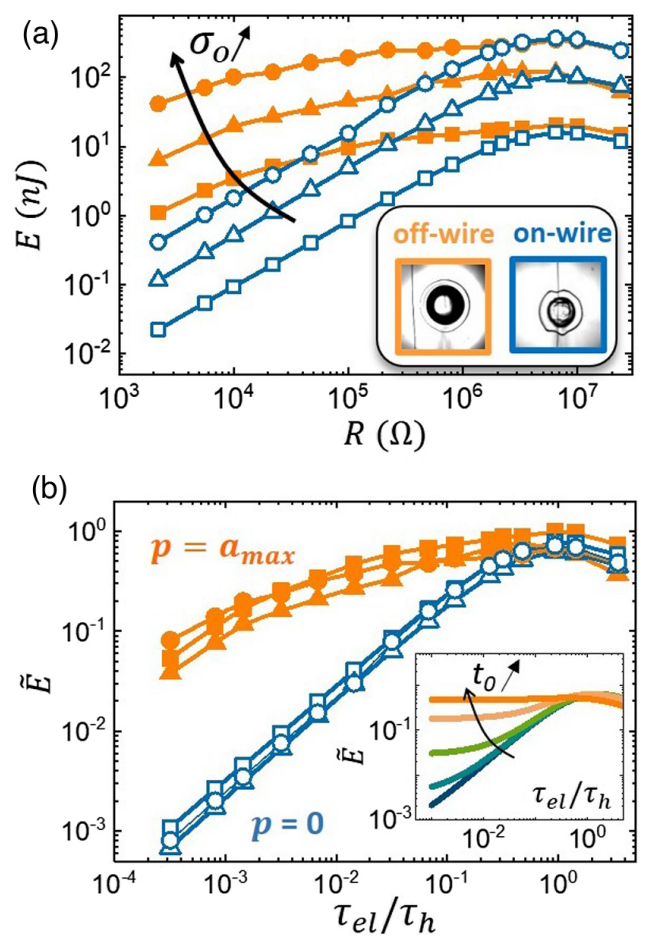

FIG. 4. Comparison of on-wire (blue) vs off-wire impact (orange) for variable load resistance. (a) Harvested energy $E$ per drop vs load resistance for variable charge densities $\left(\alpha=30^{\circ}\right.$; squares: $\quad 0.07 \mathrm{mC} / \mathrm{m}^{2}$; triangles: $0.18 \mathrm{mC} / \mathrm{m}^{2}$; circles: $0.35 \mathrm{mC} / \mathrm{m}^{2}$. Blue: drop impact on wire; orange: drop impact off-wire). (b) Normalized energy $\left(E / E_{0}\right)$ vs $\tau_{\mathrm{el}} / \tau_{h}$ [same data as (c)]. Inset: $E / E_{0}$ vs $\tau_{\mathrm{el}} / \tau_{h}$ for variable $0<t_{0}<\tau_{h}$ based on numerical solution of Eq. (1) (see Supplemental Material [28], Sec. IV and Fig. S6).

reports of $0.01 \%[6,7]$ and is only approached by the very recent study by Xu et al. [6], who used the same electrode configuration as proposed by us.

Improvements of the EWCI method are expected to enable charge densities beyond $1 \mathrm{mC} / \mathrm{m}^{2}$. In this case, energy conversion efficiencies exceeding $10 \%$ will be possible. Under these conditions, however, we expect the electrical energy extraction to affect the drop dynamics. From an applied perspective, however, one should notice that recovering appreciable amounts of energy with such technologies requires rather intense streams of droplets. For instance, recovering $10 \mathrm{~W} / \mathrm{m}^{2}$ requires the mechanical power (and thus the droplet flux) of a typical bathroom shower with $W_{\text {mech }}=5 \times 10^{5} \mathrm{~Pa} \times 10(L / \mathrm{min}) \approx 100 \mathrm{~W}$ spread over $1 \mathrm{~m}^{2}$.

In summary, our simultaneous measurements of drop spreading and electrical response provide a quantitative description of the energy harvesting process upon drop impact and allow us to identify conditions of optimum energy harvesting that do not coincide with conditions for maximum peak current and power. Thanks to our advanced electrode geometry and the high and stable surface charge densities based on EWCI, we achieved record energy conversion efficiencies in the low percent range. The derived scaling law indicates the importance of matching the timescales of the external circuit and the hydrodynamic spreading process, as well provides device design criteria for energy conversion efficiencies beyond $10 \%$.

H. W. and G.Z. acknowledge support from National Key R\&D Program of China (No. 2016YFB0401501), Program for Chang Jiang Scholars and Innovative Research Teams in Universities (No. IRT_17R40), Program for Guangdong Innovative and Entrepreneurial Teams (No. 2019BT02C241), Science and Technology Program of Guangzhou (No. 2019050001), Guangdong Provincial Key Laboratory of Optical Information Materials and Technology (No. 2017B030301007), National Center for International Research on Green Optoelectronics (IRGO), MOE International Laboratory for Optical Information Technologies, and the 111 Project.

*Corresponding author.

haowu@m.scnu.edu.cn

Corresponding author.

guofu.zhou@m.scnu.edu.cn

Corresponding author.

f.mugele@utwente.nl

[1] Y. Liu, L. Moevius, X. Xu, T. Qian, J. M. Yeomans, and Z. Wang, Nat. Phys. 10, 515 (2014).

[2] J. C. Bird, R. Dhiman, H.-M. Kwon, and K. K. Varanasi, Nature (London) 503, 385 (2013).

[3] H. Lhuissier, C. Sun, A. Prosperetti, and D. Lohse, Phys. Rev. Lett. 110, 264503 (2013).

[4] D. Richard, C. Clanet, and D. Quéré, Nature (London) 417, 811 (2002).

[5] J. De Ruiter, R. Lagraauw, D. Van Den Ende, and F. Mugele, Nat. Phys. 11, 48 (2015).

[6] W. Xu et al., Nature (London) 578, 392 (2020).

[7] Z. H. Lin, G. Cheng, S. Lee, K. C. Pradel, and Z. L. Wang, Adv. Mater. 26, 4690 (2014).

[8] J. de Ruiter, J. M. Oh, D. van den Ende, and F. Mugele, Phys. Rev. Lett. 108, 074505 (2012).

[9] W. Tang, B. D. Chen, and Z. L. Wang, Adv. Funct. Mater. 29, 1901069 (2019).

[10] J. H. Lee, S. Kim, T. Y. Kim, U. Khan, and S.-W. Kim, Nano Energy 58, 579 (2019).

[11] Z. H. Lin, G. Cheng, L. Lin, S. Lee, and Z. L. Wang, Angew. Chem. Int. Ed. 52, 12545 (2013).

[12] Y. Wang, S. Gao, W. Xu, and Z. Wang, Adv. Funct. Mater. 30, 1908252 (2020).

[13] F. Mugele and J. Heikenfeld, Electrowetting: Fundamental Principles and Practical Applications (John Wiley \& Sons, Weinheim, Germany, 2018).

[14] F. Mugele and J.-C. Baret, J. Phys. Condens. Matter 17, R705 (2005).

[15] T. Krupenkin and J. A. Taylor, Nat. Commun. 2, 448 (2011).

[16] A. Z. Stetten, D. S. Golovko, S. A. Weber, and H.-J. Butt, Soft Matter 15, 8667 (2019).

[17] S. Matsusaka, H. Maruyama, T. Matsuyama, and M. Ghadiri, Chem. Eng. Sci. 65, 5781 (2010). 
[18] K. M. Forward, D. J. Lacks, and R. M. Sankaran, Phys. Rev. Lett. 102, 028001 (2009).

[19] C. Zhang, L. Zhou, P. Cheng, X. Yin, D. Liu, X. Li, H. Guo, Z. L. Wang, and J. Wang, Appl. Mater. Today 18, 100496 (2020).

[20] J. Wang, C. Wu, Y. Dai, Z. Zhao, A. Wang, T. Zhang, and Z. L. Wang, Nat. Commun. 8, 88 (2017).

[21] R. Zimmermann, U. Freudenberg, R. Schweiß, D. Küttner, and C. Werner, Curr. Opin. Colloid Interface Sci. 15, 196 (2010).

[22] R. Zimmermann, N. Rein, and C. Werner, Phys. Chem. Chem. Phys. 11, 4360 (2009).

[23] L. Xu, T.Z. Bu, X. D. Yang, C. Zhang, and Z. L. Wang, Nano Energy 49, 625 (2018).

[24] N. Agmon, H. J. Bakker, R. K. Campen, R. H. Henchman, P. Pohl, S. Roke, M. Thämer, and A. Hassanali, Chem. Rev. 116, 7642 (2016).

[25] G. Zhou, H. Wu, and F. Mugele, China Patent, CN110572071A, 2019, https://patentimages.storage .googleapis.com/8b/3e/73/664664152f3dc2/CN110572071A .pdf.

[26] H. Wu, R. Dey, I. Siretanu, D. van den Ende, L. Shui, G. Zhou, and F. Mugele, Small 16, 1905726 (2020).

[27] H. Wu, F. Mugele, and G. Zhou, China Patent, CN110665552A, 2019, https://patentimages.storage .googleapis.com/22/5a/13/ad02dc159d7c4a/CN110665552A .pdf.
[28] See Supplemental Material at http://link.aps.org/ supplemental/10.1103/PhysRevLett.125.078301 for details of the substrate fabrication and surface charge measurements, which includes Refs. [13,26,29-31].

[29] H. Wu, B. Tang, R. A. Hayes, Y. Dou, Y. Guo, H. Jiang, and G. Zhou, Materials 9, 707 (2016).

[30] A. G. Banpurkar, Y. Sawane, S. M. Wadhai, C. Murade, I. Siretanu, D. van den Ende, and F. Mugele, Faraday Discuss. 199, 29 (2017).

[31] H. Verheijen and M. Prins, Langmuir 15, 6616 (1999).

[32] S. van der Walt, J. L. Schönberger, J. Nunez-Iglesias, F. Boulogne, J. D. Warner, N. Yager, E. Gouillart, and T. Yu, The Scikit-image contributors, PeerJ PrePrints 2:e336v2, https://doi.org/10.7287/peerj.preprints.336v2 (2014).

[33] Note that in reality, the electrons do not need to actually get transferred from the wire to the drop via a Faradaic reaction. A more sophisticated electric circuit analysis shows that polarization of the wire-electrolyte and the electrolyte-polymer interface is enough to achieve perfect screening.

[34] B. K. Yun, H. S. Kim, Y. J. Ko, G. Murillo, and J. H. Jung, Nano Energy 36, 233 (2017).

[35] I. V. Roisman, R. Rioboo, and C. Tropea, Proc. Math. Phys. Eng. Sci. 458, 1411 (2002).

[36] Y. Suzuki, IEEJ Trans. Electr. Electron. Eng. 6, 101 (2011).

[37] P. D. Mitcheson, E. M. Yeatman, G. K. Rao, A. S. Holmes, and T. C. Green, Proc. IEEE 96, 1457 (2008). 